\title{
How does attention spread across objects oriented in depth?
}

\author{
IRENE REPPA \\ Swansea University, Swansea, Wales \\ DARYL Fougnie \\ Vanderbilt University, Nashville, Tennessee \\ AND \\ William C. SCHMIDT \\ University at Buffalo, State University of New York, Buffalo, New York
}

\begin{abstract}
Previous evidence suggests that attention can operate on object-based representations. It is not known whether these representations encode depth information and whether object depth, if encoded, is in viewer- or objectcentered coordinates. To examine these questions, we employed a spatial cuing paradigm in which one corner of a 3-D object was exogenously cued with $75 \%$ validity. By rotating the object in depth, we can determine whether validity effects are modulated by 2-D or 3-D cue-target distance and whether validity effects depend on the position of the viewer relative to the object. When the image of a 3-D object was present (Experiments 1A and $1 \mathrm{~B}$ ), validity effects were not modulated by changes in 2-D cue-target distance, and shifting attention toward the viewer led to smaller validity effects than did shifting attention away from the viewer. When there was no object in the display (Experiments 2A and 2B), validity effects increased linearly as a function of 2-D cue-target distance. These results demonstrate that attention spreads across representations of perceived objects that encode depth information and that the object's orientation in depth is encoded in viewer-centered coordinates.
\end{abstract}

The properties of selective attention in both 2-D and 3-D space have been extensively studied using exogenous spatial cuing paradigms in which one display location is cued before an ensuing target (e.g., Downing \& Pinker, 1985; Posner, 1980). In 2-D space, when the target appears within half a second or less from the cue onset, participants are more accurate and faster to respond when the target appears at the cued location (valid cue trials) than when it appears at unexpected or invalidly cued locations (invalid cue trials). In addition, the performance difference between valid and invalid cue trials, termed a validity effect, can depend on the spatial distance between the cue and the target, with longer distances leading to larger validity costs (e.g., Egly \& Homa, 1991; Posner, 1980).

Studies of selective attention in 3-D space have also shown that attention can be allocated to distinct locations in depth, with costs of attention arising when attention shifts from a location in one depth plane to a location in another depth plane (e.g., Andersen, 1990; Andersen \& Kramer, 1993; Arnott \& Shedden, 2000; Atchley, Kramer, Andersen, \& Theeuwes, 1997; Downing \& Pinker, 1985; Gawryszewski, Riggio, Rizzolatti, \& Umiltà, 1987; He \& Nakayama, 1995; Theeuwes, Atchley, \& Kramer, 1998). These studies show that depth is encoded in our representation of space and that this representation can be ac- cessed by selective attention. Furthermore, some studies on selective attention in 3-D space have shown that shifting attention away from the viewer leads to larger validity effects than does shifting attention toward the viewer, suggesting that 3-D space is represented in viewer-centered coordinates (e.g., Andersen, 1990; Andersen \& Kramer, 1993; Arnott \& Shedden, 2000; Downing \& Pinker, 1985; Gawryszewski et al., 1987).

In addition to evidence that selective attention operates across empty space, there is considerable evidence that the presence of objects can alter the way selective attention is allocated in both 2-D and 3-D space. Studies using objects (e.g., rectangles) extending to different locations in two dimensions have shown that whole objects can be selected (e.g., Baylis \& Driver, 1992; Egly, Driver, \& Rafal, 1994), as can individual object parts (e.g., Leek, Reppa, \& Tipper, 2003; Reppa \& Leek, 2003, 2006; Vecera, Behrmann, \& Filapek, 2001; Vecera, Behrmann, \& McGoldrick, 2000). Differences in validity effects between two locations that are part of an object are observed to be smaller than those between two locations on different objects, even if the spatial distance is equivalent (e.g., Egly et al., 1994). These findings show that shifts of attention from one location to another can be reduced if those locations are part of the same object (e.g., Duncan, 1984; Egly et al., 1994) and

I. Reppa, i.reppa@swansea.ac.uk, or D. Fougnie, d.fougnie@vanderbilt.edu 
that the presence of objects can alter the perceptual organization of the visual scene and, subsequently, the shifts of attention to locations in that scene (e.g., Robertson \& Kim, 1999). Typically, such findings are taken to support the notion that attention can be object based - that it can select representations of distinct objects and object parts from the visual scene.

Similar observations have been made for objects (e.g., Atchley \& Kramer, 2001; Gibson \& Egeth, 1994; Umiltà, Castiello, Fontana, \& Vestri, 1995) and perceived surfaces (e.g., He \& Nakayama, 1995) extending in depth. For instance, He and Nakayama's Experiment 3 showed that validity costs for targets located on a stereo-defined surface were equally large, irrespective of the amount of disparity (i.e., distance in depth), suggesting that attention can operate on perceived surfaces extending in depth. Similarly, in a divided attention task, using realistic objects, Atchley and Kramer found that targets appearing at two different depths were detected faster if they belonged to the same object than if they belonged to two different objects. These findings suggest that the presence of an object extending in depth can also eliminate effects of switching attention in depth.

Therefore, so far, studies on selective attention in 3-D space show that information about depth (whether two locations are in the same or a different depth) is encoded in the representation of space in viewer-centered coordinates and that it can influence selective attention. Furthermore, evidence from studies in which objects extending in 2-D or in 3-D space were used shows that the cost of switching attention from one location to another are eliminated when those locations appear within the same object or on the same surface (e.g., Atchley \& Kramer, 2001; Egly et al., 1994; He \& Nakayama, 1995).

One theoretically important issue that is often neglected is the nature of object shape representations that underlie any observed object-based attention effects. This issue pertains to the shape primitives that underlie such representations (i.e., whether they are part-based or holistic; e.g., Leek et al., 2003; Reppa \& Leek, 2003, 2006; Vecera et al., 2001; Vecera et al., 2000). It also pertains to whether the object representations underlying object-based attention effects are 2-D or 3-D (e.g., Müller \& O'Grady, 2009). In the present study, we examined the second issue: Do the representations of selected 3-D objects contain depth information? Currently, neither evidence from space-based attention studies nor evidence from object-based attention studies can offer an answer to this question. First, knowing that depth is encoded in our representation of (empty) space and that this representation can modulate attention does not inform us on whether depth is encoded in the representation of the space within an object. One possibility is that space within an object is represented by principles similar to those for empty space that is not constrained by object contours, and therefore, as with empty space, depth will also be encoded as part of the representation of the space within an object. Another possibility is that space within an object is represented differently from space outside objects, therefore leaving the question open of whether depth is represented in the representation of the space within objects-in other words, whether depth is encoded in the representations of objects selected by attention.

Second, knowing that attention can select objects or object surfaces that either lie along a single depth plane or extend in depth cannot tell us whether depth is encoded as part of the selected object representation. That is, the elimination of selection costs due to the presence of an object surface extending in depth could mean that depth is not encoded in the representation of the object but, instead, that selective attention operates on a 2-D representation of the attended object surface, regardless of whether it extends in depth (e.g., Müller \& O'Grady, 2009). Alternatively, it could mean that depth is encoded in the representation of the selected object and attention operates on a representation of the object that includes full depth information (e.g., a 3-D object representation). The latter possibility remains untested. ${ }^{1}$ Furthermore, if the orientation of the object in depth is encoded in the object's representation, it may be represented relative to the viewer, yielding a representation of the 3-D object that is viewer centered, or depth may be represented relative to one of the object's global attributes (and entirely independently of the viewer), such as its axis of elongation, yielding a fully $3-\mathrm{D}$ object-centered representation of the object.

The issue of whether and how depth is encoded in the representation of objects is also relevant and debated within current theories of object recognition. For instance, one important question in object recognition is whether the relationship between the object and the viewer is explicitly encoded in the representations of 3-D objects. Some accounts propose that objects are represented as configurations of fully 3-D viewpoint-invariant parts coded relative to a global attribute of the object, independently of the viewing position (such as the object's axis of symmetry or elongation; e.g., Biederman, 1987; Biederman \& Gerhardstein, 1995; Marr \& Nishihara, 1978). Other accounts, although they agree that objects are represented as 3-D models, propose that those representations also contain some viewpoint-specific information about depth (e.g., Ullman, 1989). Finally, other object recognition accounts propose that 3-D object shape is represented by 2 -D viewpoint-dependent features, such as outline contours (e.g., Tarr \& Bülthoff, 1998; Tarr \& Pinker, 1989) or surfaces (e.g., Leek, Reppa, \& Arguin, 2005; Tarr \& Bülthoff, 1998) that are coded relative to the viewer and have little or no depth information (e.g., Bülthoff, Edelman, \& Tarr, 1995).

Our aim in the present study was to examine whether object-based attention is mediated by representations that encode the orientation of the object in depth and whether the object's orientation in depth, if encoded, is in viewercentered or object-centered coordinates. In Experiments $1 \mathrm{~A}$ and $1 \mathrm{~B}$, one of four corners of the front plane of a hollow 3-D square was cued (Figures 1A and 1B) and participants made speeded detection responses to targets at the same location as the cue (valid trials) or at one of the corners adjacent to the cue (invalid trials). Critically, the square appeared either unrotated or rotated in depth 
A

Experiment 1A

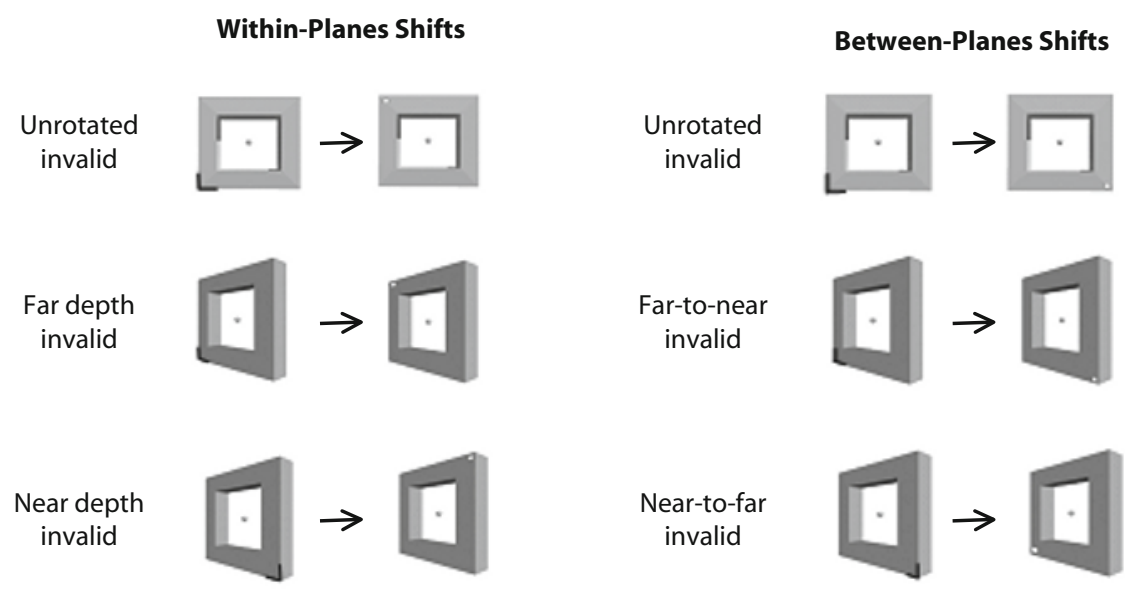

B

Experiment 1B

Within-Planes Shifts
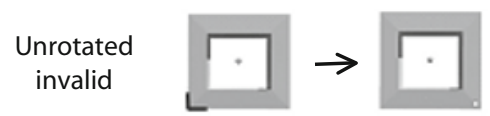

Far depth

invalid

Near depth

invalid
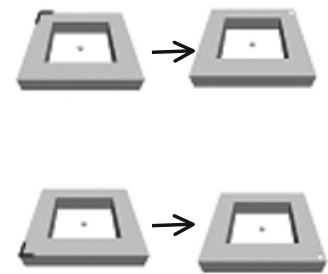

Near-to-far invalid
Between-Planes Shifts

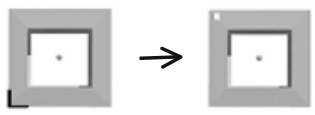

Far-to-near invalid
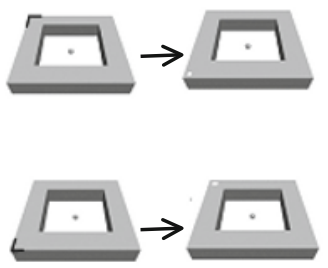

Figure 1. (A) The six types of invalid conditions of Experiment $1 \mathrm{~A}$ shown only for the frontand left-rotated views (a right-rotated view was also used but is not shown here). (B) The six types of invalid conditions of Experiment 1B shown only for the front- and backward-rotated views (the forward-rotated view is not shown here). The dark $L$-shape was the cue, and the white box was the target. Color information has been discarded here, but see the Method section for details. For Experiments $2 \mathrm{~A}$ and 2B, the object was removed, but all other stimuli and cue-target configurations remained the same.

by $45^{\circ}$. Although rotation affected the 2 -D cue-target distances, perceived 3-D distances within the object were the same. Because of the rotation, the 2-D extent at the far end of the rotated object was shorter, and the near end longer, than when the object was unrotated. Horizontally, the 2-D extent of the surface was shorter in depth when the object was rotated than when it was unrotated. In Experiments 2A and 2B, the object was removed from the display, but all cue and target conditions remained the same.

This design allowed us to investigate two issues regarding the representations mediating the spread of attention across the image of a 3-D object. First, we examined whether attention spreads across the 2-D extent of the object or whether it encodes depth-by comparing attention shifts along the near end of the object with those along the far end. If depth is encoded in the representation of the object on which attention operates, this would suggest that 3-D, but not 2-D, cue-target distance would mediate response latencies when the object is present (Experiments $1 \mathrm{~A}$ and $1 \mathrm{~B})$. In contrast, 2-D cue-target distance should mediate response latencies when object contours are absent and object-based representations are inoperative (Experiments 2A and 2B).

The second question was whether the object's orientation in depth, if encoded in the object representation, is in object-centered or viewer-centered coordinates. If the object's orientation in depth is encoded in viewer-centered coordinates, shifting attention toward the viewer could lead to faster response latencies than would shifting attention away from the viewer, similar to how visual attention operates on unparsed space (e.g., Andersen, 1990; Andersen \& Kramer, 1993; Arnott \& Shedden, 2000; Gawryszewski et al., 1987). In contrast, if the object's orientation 
in depth is encoded in object-centered coordinates, there would necessarily be no difference between attention shifts toward or away from the viewer.

\section{EXPERIMENTS 1A AND 1B}

\section{Method}

The methodological details were the same for Experiments 1A and $1 \mathrm{~B}$ unless otherwise stated.

\section{Participants}

Twenty undergraduate students (Experiment 1A) and a different sample of 20 undergraduate and postgraduate students (Experiment 1B) at Swansea University were given course credit to participate. All of the participants had normal or corrected-to-normal vision and were naive as to the purpose of the experiment.

\section{Apparatus and Stimuli}

The stimuli (Figures 1A and 1B) were displayed on a computer monitor at a $57-\mathrm{cm}$ viewing distance. All of the stimuli appeared against a black background and were viewed monocularly to reduce discrepancy between the perceived three-dimensionality of the object and the two-dimensionality of the computer screen. A hollow, 3-D rendered square was created in Strata 3-DPro, lit from an external source. The square was rendered in two positions: either unrotated or rotated in depth by $45^{\circ}$ of visual angle. When the object rotated, the light source did not rotate with the object. In Experiment 1A, the square was rotated around its $y$-axis to the left or to the right. In Experiment 1B, it was rotated along its $x$-axis forward or backward. When the square was unrotated, its front surface measured $7.5^{\circ} \times 7.5^{\circ}$. When the square was rotated, its extent in depth was $4.5^{\circ}$, its extent around the perceived-near depth plane was $8.5^{\circ}$, and its extent along the far depth plane was $6.5^{\circ}$. The fixation sphere's diameter was $0.4^{\circ}$. The cue was a red L-shape with $1.7^{\circ}$ line segments, and the target was a white square $\left(0.5^{\circ} \times 0.5^{\circ}\right)$. Once created with the above measurements, the cue and target were deformed so that they appeared as part of the object's surface at each orientation. Cue visibility was calculated using the Weber contrast, with the stimulus luminance being the luminance of the cue $\left(7.22 \mathrm{~cd} / \mathrm{m}^{2}\right)$ and the background luminance being the luminance of the object surface $\left(17.13 \mathrm{~cd} / \mathrm{m}^{2}\right.$ when unrotated and $13.94 \mathrm{~cd} / \mathrm{m}^{2}$ when rotated). Cue visibility when the object was unrotated was $C_{\mathrm{W}}=$ -.58 and when the object was rotated was $C_{\mathrm{W}}=-.48$

\section{Design and Procedure}

Cue-target validity and target depth were manipulated within subjects. Cue-target validity had three levels: valid, the cue and the target appeared at the same location on the object; invalid withinplanes, the cue and the target appeared along the same depth plane (e.g., near); and invalid between-planes, the cue appeared on one depth plane (e.g., near) and the target appeared on the other (e.g., far). Target depth also had three levels: unrotated, the target appeared on any corner of the unrotated square; far, the target appeared on any of the two far corners of the left- or right-rotated square; and near, the target appeared on any of the two near corners of the rotated square. The combination of the two variables gave rise to three valid (valid unrotated, valid far, valid near) and six invalid types of trial. The invalid trials were categorized (Figure 1A) as a function of target location in depth relative to the viewer (near, far, or unrotated) and whether the cue and target appeared on the same or a different depth plane (between- vs. within-planes shifts). For the rotated objects, when the cue and target appeared on different depth planes (between-planes shifts), the invalidly cued near and far targets were labeled far-to-near and near-to-far, respectively. When the cue and target appeared within the same plane (within-planes shifts), the invalidly cued targets were termed far or near, depending on their perceived location relative to the viewer. For the unrotated objects, in the service of comparison with the between- and within-planes shift conditions when the object was rotated, horizontal and vertical shifts were labeled as follows. In Experiment 1A, horizontal invalid trials were labeled between-planes shifts, and vertical invalid trials were labeled within-planes shifts. In Experiment $1 \mathrm{~B}$, the labeling was reversed, and now horizontal invalid trials were labeled within-planes shifts, and vertical invalid trials were labeled between-planes shifts.

In Experiment 1A, the object appeared unrotated in 33\% of the trials and was rotated around the $y$-axis to the left or to the right with equal probability in the remaining $66 \%$ of the trials. Similarly, in Experiment 1B, the object appeared unrotated in $33 \%$ of the trials and was rotated around the $x$-axis forward or backward with equal probability in the remaining $66 \%$ of the trials. Object rotation (unrotated, rotated) was randomized throughout the trials in both experiments. In each experiment, there were 432 trials in total, and in 48 of those trials (11\%), no target appeared (catch trials). The target appeared at the same location as the cue in $75 \%$ (288) of the trials. For the remaining $25 \%$ (96; invalid trials), the target appeared at a corner of the object either vertically or horizontally opposite from the cue.

Each trial began with the fixation sphere at screen center, followed by the image of a 3-D square 1,000 msec later. Both the fixation sphere and the square remained visible until the trial ended. After 1,000 msec from the onset of the square, the cue appeared randomly and with equal probability on one of the four corners of the front face of the square and remained for $100 \mathrm{msec}$. After $200 \mathrm{msec}$ from cue offset, the target (or nothing) appeared for $1,500 \mathrm{msec}$ or until a response was registered. The participants were instructed to press the space bar on the keyboard as soon as they detected the target and to withhold their response if no target appeared. A 500$\mathrm{Hz}$ beep sounded if a keypress was detected in the catch trials. The instructions emphasized the importance of using the cue to allocate attention covertly, while maintaining eye fixation on the sphere and avoiding eye movements toward any other events on the screen. A chinrest was used to keep the participants' head from moving.

\section{Experiment 1A Results}

Responses for catch trials $(2.2 \%)$, responses faster than $150 \mathrm{msec}(6 \%)$, which were considered anticipatory, and responses slower than $1,000 \mathrm{msec}(0.6 \%)$, which were considered too slow for this task (e.g., Vecera, 1994), were not included in the analyses. A 3 (validity: valid, invalid between planes, invalid within planes) $\times 3$ (target depth: unrotated, far, near) repeated measures ANOVA, with correct mean response time (RT) as the dependent measure, revealed significant main effects of validity $[F(2,38)=$ $10.97, p=.0001]$ and target depth $[F(2,38)=8.50, p=$ $.001]$. The validity $\times$ target depth interaction was not significant $[F(4,76)<1, p>.05]$. Cell means are reported in Table 1.

\section{Invalid RT Analysis}

The effect of target depth on the mean RTs of invalid trials was examined in order to address the two issues under investigation: whether RT performance is mediated by 2-D or 3-D cue-target distance, and whether the object's orientation in depth, if encoded in the object representation, is encoded in object-centered or viewercentered coordinates. Here, we report two analyses of invalid RTs. In the first set of analyses, we examined between-planes shifts for the rotated objects (i.e., when the cue and target appeared at different depth planes on the object). For comparison purposes, in this analysis, we included the horizontal shifts along the unrotated objects, which we labeled between-planes shifts (for details, see the Design section). In the second set of analyses, we examined within-planes shifts for the rotated objects (i.e., 
Table 1

Mean Response Times (RTs) and Standard Deviations for Each Validity and Target Depth Condition

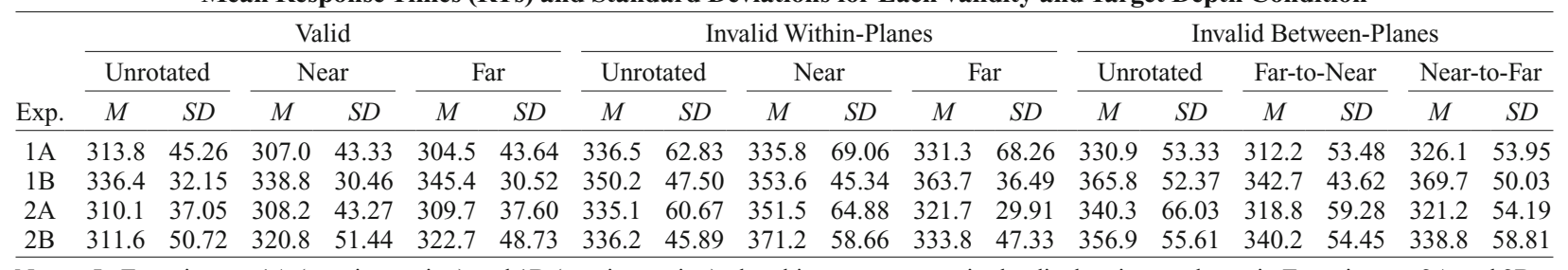

Note-In Experiments 1A ( $y$-axis rotation) and 1B ( $x$-axis rotation), the object was present in the display; it was absent in Experiments $2 \mathrm{~A}$ and $2 \mathrm{~B}$.

when the cue and target appeared along the same depth plane of the object). Again, for comparison purposes, we included the vertical cue-target attention shifts along the unrotated objects, which we labeled within-planes shifts (see the Design section).

Between-planes shifts. A one-way repeated measures ANOVA showed a significant effect of target depth (unrotated, far-to-near, near-to-far) on invalid between-planes RTs $[F(2,38)=6.03, p=.005]$. Far-to-near RTs were significantly shorter than unrotated RTs $[t(19)=2.92$, $p=.009]$ and near-to-far RTs $[t(19)=2.61, p=.01]$, revealing a viewer-centered orienting of attention along the surface of the perceived 3-D object. Unrotated and near-to-far RTs did not differ $[t(19)<1, p>.05]$, despite their differences in 2-D cue-target distance.

Within-planes shifts. A one-way repeated measures ANOVA showed no significant effect of target depth (unrotated, near-to-far, far-to-near) on invalid within-planes $\operatorname{RTs}[F(2,38)<1, p>.05]$, indicating that how attention spreads across a representation of the object's surface is not affected by changes in 2-D distance.

\section{Valid RTs and Validity Effects Analysis}

Validity effects were analyzed to establish that the RT data pattern reported above reflects differences in shifting attention, as opposed to differences influenced by attending to different depths, even without the need to shift attention (i.e., as in the case of valid trials). Indeed, the effect of target depth (unrotated, far, near) on valid trial RTs was significant $[F(2,38)=6.35, p=.004]$. Therefore, validity effects were calculated by subtracting the valid RTs with matching target depth from the invalid RTs of each of the six invalid conditions (e.g., between-planes far validity effect $=$ invalid between-planes far RT - valid far RT). The validity effects for Experiment 1A are shown in Figure $2 \mathrm{~A}$. Between-planes validity effects were significant for the unrotated $(M=17.0 \mathrm{msec}, S D=31.8)[t(19)=$ $2.39, p=.02]$ and the near-to-far $(M=21.6 \mathrm{msec}, S D=$ 25.3) $[t(19)=3.82, p=.001]$ conditions, but not for the far-to-near condition $(M=5.2 \mathrm{msec}, S D=21.7)[t(19)=$ $1.06, p>.05]$. All within-planes validity effects were significant [unrotated, $M=22.6 \mathrm{msec}, S D=32.7, t(19)=$ $3.09, p=.006$; far depth, $M=26.7 \mathrm{msec}, S D=40.4$, $t(19)=-2.96, p=.008$; near depth, $M=28.8 \mathrm{msec}$, $S D=42.3, t(19)=-3.04, p=.007]$.

We examined the effect of target depth on validity effects separately for between- and within-planes shifts. To anticipate, the pattern of results for the validity effects mirrored the pattern in the RT data for both types of shift.
Between-planes shifts. The between-planes validity effects mirrored the between-planes RT data. A one-way repeated measures ANOVA on validity effects showed a significant effect of target depth $[F(2,38)=3.51, p=.02]$. Validity effects did not differ between unrotated and nearto-far shifts $[t(19)< \pm 1, p>.05]$, but the validity effects in the far-to-near condition were significantly smaller than those in both the unrotated $[t(19)=2.01, p=.04]$ and the near-to-far $[t(19)=2.40, p=.02]$ conditions.

Within-planes shifts. A one-way repeated measures ANOVA on validity effects revealed no significant main effect of target depth $[F(2,38)<1, p>.05]$, replicating the RT pattern for within-planes shifts.

\section{Experiment 1B Results}

For the catch trials $(1 \%)$, responses faster than $150 \mathrm{msec}(2.5 \%)$ and slower than $1,000 \mathrm{msec}(0.5 \%)$ were not included in the analyses. Furthermore, the data from 1 participant were not included in the analysis because of unusually high RTs (more than $2 S D$ s from the group mean). A 3 (validity: valid, invalid between planes, invalid within planes) $\times 3$ (target depth: unrotated, far, near) repeated measures ANOVA, with correct mean RT as the dependent measure, revealed a significant main effect of validity $[F(2,36)=6.54, p=.004]$. The main effect of target depth was not significant $[F(2,36)<1, p>.05]$, but there was a significant validity $\times$ target depth interaction $[F(4,72)=5.75, p=.0001]$. Table 1 shows the cell means.

\section{Invalid RT Analysis}

As in Experiment 1A, invalid trials for Experiment 1B were categorized as a function of target location relative to the viewer (near, far, or unrotated) and whether the cue and target appeared on the same or a different depth plane (between and within planes). For the rotated objects, when the cue and the target appeared within the same plane, the invalidly cued targets were termed far or near, depending on their perceived location along the object relative to the viewer. When the cue and the target appeared on different depth planes, the invalidly cued near and far targets were labeled as far-to-near and near-to-far; respectively. For the unrotated objects, unlike in Experiment 1A, vertical shifts were now labeled between-planes shifts and horizontal shifts were labeled within-planes shifts (for comparison with the rotated conditions).

Between-planes shifts. A one-way repeated measures ANOVA showed a significant effect of target depth (unrotated vs. near-to-far vs. far-to-near) on invalid between- 


\section{A Experiment 1A: Object Present}

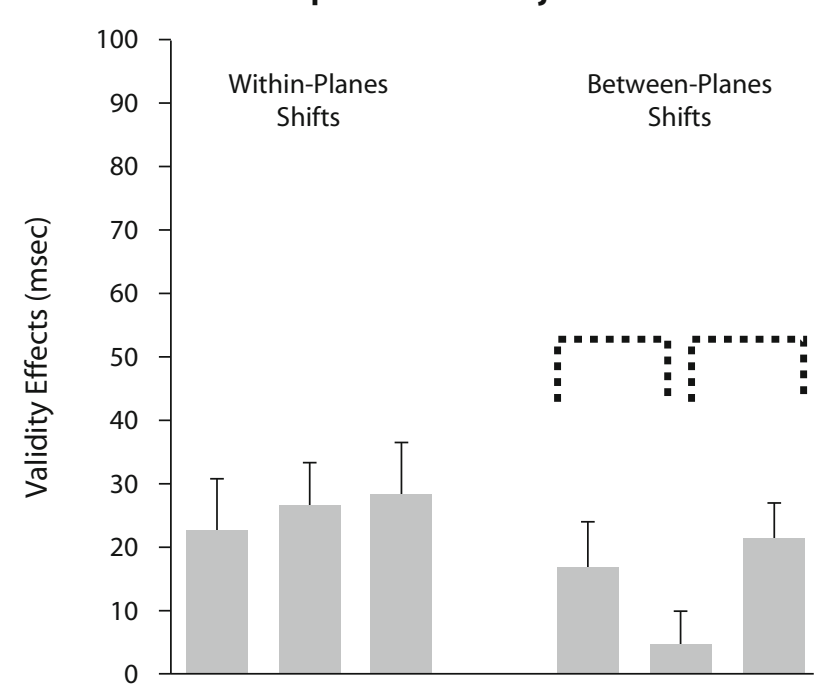

\section{Experiment 2A: Object Absent}

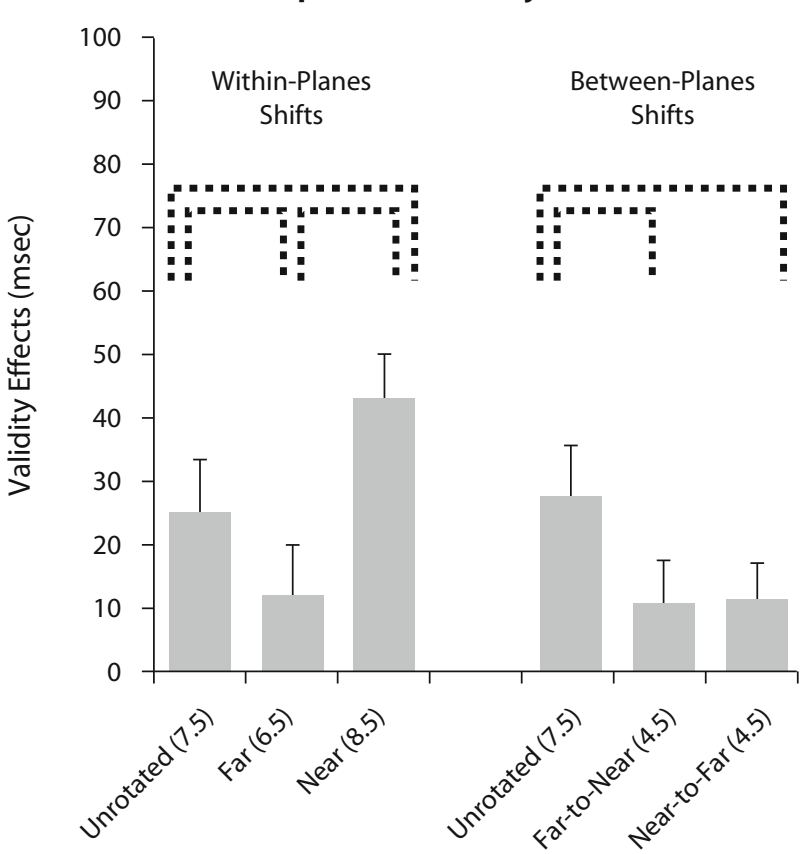

B Experiment 1B: Object Present

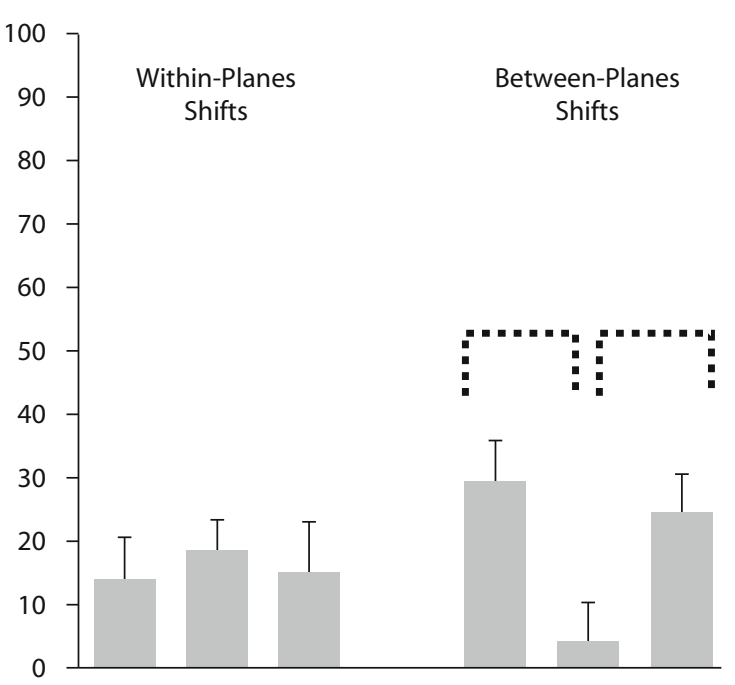

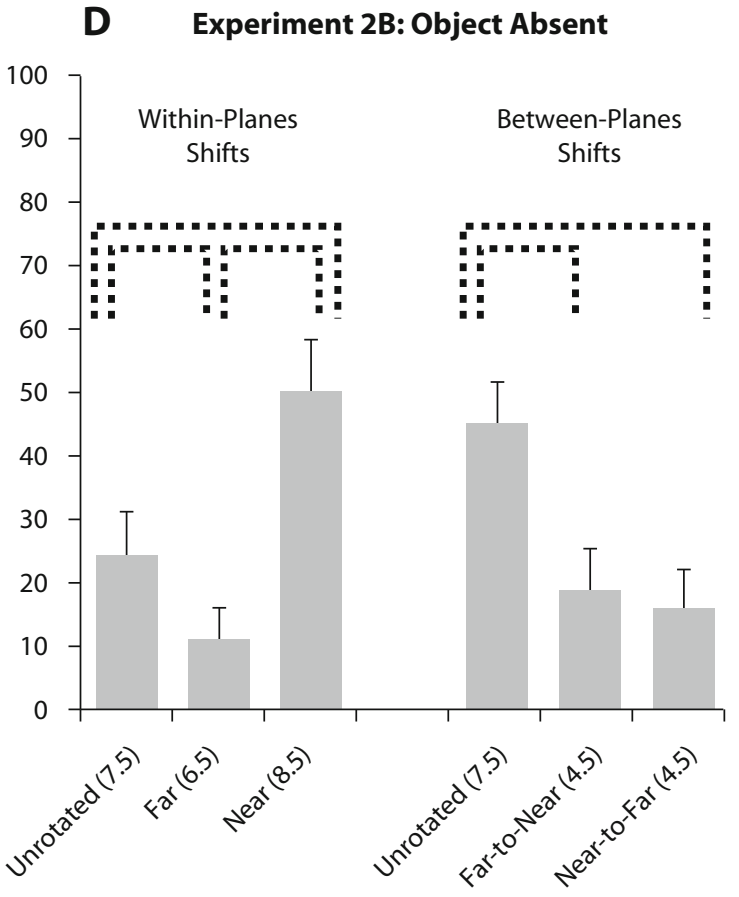

Figure 2. Validity effects (invalid RT - valid RT) for each invalid condition in each of the three rotation conditions in Experiments 1 A (object present, $y$-axis rotation) and $1 \mathrm{~B}$ (object present, $x$-axis rotation) and Experiments $2 \mathrm{~A}$ (object absent, $y$-axis rotation) and $2 \mathrm{~B}$ (object absent, $\boldsymbol{x}$-axis rotation). Numbers in parentheses along the $\boldsymbol{x}$-axis denote the distance between the cue and the target on the computer screen in degrees of visual angle. Error bars represent the standard errors of the means. Significant differences $(p<.05)$ are illustrated by connecting lines.

planes $\mathrm{RTs}[F(2,36)=4.70, p=.01]$. The far-to-near RTs were significantly faster than the unrotated RTs $[t(18)=$ 2.28, $p=.03]$ and the near-to-far RTs $[t(18)=2.98, p=$ $.008]$, indicating a viewer-centered spread of attention along the surface of the perceived 3-D object. Unrotated and near-to-far RTs did not differ $[t(18)< \pm 1, p>.05]$.

Within-planes shifts. A one-way repeated measures ANOVA on invalid within-planes RTs with target depth as the repeated measure (unrotated, far, near) showed no significant effect $[F(2,36)=1.40, p>.05]$.

\section{Valid RTs and Validity Effects Analysis}

The effect of target depth (unrotated, far, near) on valid RTs was significant $[F(2,36)=3.59, p=.04]$; therefore, validity effects for the six invalid conditions were calculated by subtracting the appropriate valid RT from the 
invalid RT for each condition. Experiment 1B validity effects are shown in Figure 2B. The between-planes validity effects were significant for the unrotated $(M=29.3 \mathrm{msec}$, $S D=71.1)[t(18)=4.10, p=.001]$ and near-to-far $(M=$ $24.2 \mathrm{msec}, S D=30.0)[t(18)=3.52, p=.001]$ but not for the far-to-near $(M=3.8 \mathrm{msec}, S D=33.6)[t(18)< \pm 1$, $p>.05]$ RTs. All within-planes validity effects were significant [unrotated, $M=13.8 \mathrm{msec}, S D=31.2, t(18)=$ $2.00, p=.03$; far RT, $M=18.2 \mathrm{msec}, S D=18.4, t(18)=$ $4.31, p=.0001$; and near RTs, $M=14.8 \mathrm{msec}, S D=$ $34.9, t(18)=1.90, p=.04]$.

Between-planes shifts. Mirroring the RT data, a oneway repeated measures ANOVA on validity effects showed a significant effect of target depth $[F(2,36)=4.19, p=$ $.02]$. Validity effects did not differ between the unrotated and the near-to-far shifts [ $t(18)< \pm 1, p>.05]$, but the far-to-near validity effects were significantly smaller than those in both the unrotated $[t(18)=2.43, p=.02]$ and the near-to-far $[t(11)=-2.12, p=.04]$ conditions.

Within-planes shifts. A one-way repeated measures ANOVA on validity effects revealed no significant main effect of target depth $[F(2,36)<1, p>.05]$, replicating the RT pattern.

\section{Experiments 1A and 1B Discussion}

Our aim in the present study was to examine the nature of object representations that mediate object-based attention effects. In particular, we examined whether the selected object representation makes depth information explicit or whether it is 2-D. We hypothesized that, if depth information is not made explicit in the selected object representation, the 2-D cue-target distance would mediate validity effects. In contrast, if depth is made explicit, the perceived cue-target distance would mediate validity effects. Furthermore, if depth is explicit in the object representation, it could be encoded in terms of either viewer-centered or object-centered coordinates. Two main findings emerged. When attention was directed within the same plane, there were no differences in RTs or in validity effects, regardless of whether the object appeared rotated or unrotated relative to the viewer, indicating that the cost of shifting attention within any object depth plane was equivalent, despite differences in 2-D cue-target distance. This finding contributes to existing evidence that attention can spread automatically across a perceived surface that is cued, as opposed to across space as it appears on the retina (e.g., He \& Nakayama, 1995; Robertson \& Kim, 1999).

The second finding was that viewer-centered effects were observed: Far-to-near shifts were faster than nearto-far shifts. Such viewer-centered attention effects have previously been reported in studies in which space-based attention in depth - that is, attention across the space between different objects appearing at different locations in depth-has been examined using real scenes (e.g., Downing \& Pinker, 1985; Gawryszewski et al., 1987) and stereoscopic displays (e.g., Andersen \& Kramer, 1993; Arnott \& Shedden, 2000). Here, we examined attention to different locations along the surface of a single object extending in depth and observed attention operating in a viewer-centered way along an object extending in depth:
Shifting attention toward the viewer was significantly faster than shifting attention away from the viewer. Critically, these effects were observed both in the absence of differences between vertical shifts for far and near planes and in the absence of differences between horizontal shifts along the unrotated object and near-to-far shifts along the rotated object. In both cases, there were differences in the 2-D cue-target distance. Therefore, although 2-D distances failed to modulate response latencies and validity effects, the perceived 3-D layout of the object did. Incidentally, this pattern of results was replicated exactly in a different lab, using displays in which the 3-D object was seen to be rotating in depth (adding an extra, depth-from-motion cue) before the cue and target appeared on the object.

This finding, to our knowledge, is the first piece of evidence that the representation of a selected object makes depth information explicit. As such, it significantly extends previous evidence for the operation of object-based attention for objects that extend along a single depth plane (e.g., Egly et al., 1994) or objects that extend in depth (e.g., Atchley \& Kramer, 2001; He \& Nakayama, 1995, Experiment 3), by sharpening our understanding of the nature of the representations that mediate such object-based attention effects. Previous researchers examining spacebased attention in depth along space between objects have also reported that depth is encoded in the representation of visual displays (i.e., performance costs when attending to two different objects at different depths, as opposed to attending to two objects at the same depth; e.g., Atchley $\&$ Kramer, 2001) and that it is encoded in viewer-centered coordinates (i.e., differential effects between far-to-near and near-to-far attention shifts; e.g., Andersen, 1990; Andersen \& Kramer, 1993; Arnott \& Shedden, 2000; Downing \& Pinker, 1985; Gawryszewski et al., 1987). The present findings show for the first time that depth is also encoded in the representation of objects. Furthermore, depth is not encoded in object-centered coordinates that discard the viewer's position relative to the object, but in viewer-centered coordinates that take into account the relative locations of the viewer and the object.

This finding has important implications for theories of attention and theories of object recognition. It constrains hypotheses that attention spreads across 2-D representations (e.g., Müller \& O'Grady, 2009; Vecera, 1994; Vecera \& Farah, 1994), showing that selective attention can be reliably modulated by a representation of the 3-D object that makes object depth relative to the viewer explicit. The finding that object depth is encoded in viewercentered coordinates also constrains hypotheses of object recognition. More specifically, depth is encoded not in object-centered coordinates, as would be hypothesized by some theories of object recognition (e.g., Biederman, 1987; Marr \& Nishihara, 1978), but in viewer-centered coordinates, as is hypothesized by view-based theories of object recognition (e.g., Tarr \& Bülthoff, 1998; Tarr \& Pinker, 1989).

One unexpected finding was that response latencies in the invalid far-to-near condition were not significantly slower than those in the valid condition in either experi- 
ment. One possible reason for this may be the poorer representation of space during this type of attentional shift. $\mathrm{Wu}$, Ooi, and $\mathrm{He}$ (2004) found that overt visual scanning of the ground surface from far-to-near locations on the ground led to an underestimation of the distance between the target and the observers, relative to visual scanning from near-to-far locations in depth. A similar but covert visual scanning process (unlike the overt scanning in $\mathrm{Wu}$ et al., 2004) is simulated in the present cuing experiments: Covert visual scanning in depth is assumed in invalid trials, where the cue and target appear at different depths. According to the proposal of Wu et al., in trials in which the cue appears at the far end of the display and the target near the observer, the distance between the two objects is likely to be underestimated, leading to faster detection of the near target. Conversely, perceived distance between the cue and the target is likely to be more accurate when the cue appears at the near end of the object and the target at the far end. Subsequently, responses to targets in the nearto-far condition would be comparatively equivalent to those in the unrotated condition and slower than those in the far-to-near condition. Nevertheless, the lack of significant validity effects in the far-to-near condition does not detract from the finding that it was the 3-D layout of the object, as opposed to its 2-D layout, that guided the spread of selective attention across the object.

\section{EXPERIMENTS 2A AND 2B}

In Experiments 1A and 1B, attention spread across the perceived extent of a 3-D object extending in depth, and we concluded that attention was operating on an objectbased representation coded in viewer-centered coordinates. Arguably, our results may reflect the operation of a purely spatial attention mechanism that spreads differentially across the visual field, regardless of whether an object is present in the display or not, rendering our conclusions from Experiments 1A and 1B premature.

To ensure that the observed effects reflect the operation of object-based attention, as opposed to the distribution of attention in 2-D space regardless of whether an object is present, in Experiments 2A and 2B, we repeated Experiments $1 \mathrm{~A}$ and $1 \mathrm{~B}$, except that no object information (and subsequently, no depth information) was present. If, even in the absence of object information, the same pattern of results emerges as in Experiments 1A and 1B, this would imply that the object - and the depth information that it also provides - did not change the normal 2-D spatial operation of attention. In contrast, if the pattern of results in Experiments $1 \mathrm{~A}$ and $1 \mathrm{~B}$ was due to the perceptual organization of space imposed by the object, the removal of the object in Experiments 2A and 2B would produce a different pattern of results. Additionally, if a comparison between Experiments $1 \mathrm{~A}$ and $1 \mathrm{~B}$ and $2 \mathrm{~A}$ and $2 \mathrm{~B}$ reveals a significant interaction between experiment and target depth, we can be certain that the lack of an effect of target depth observed in Experiments 1A and 1B for within-planes shifts is not simply a null effect due to insufficient power. Following previous findings that visual attention spreads in visual space as a linear function of the distance between two locations (e.g., Egly \& Homa, 1991), it was predicted that RTs would vary as a linear function of cue-target distance, with longer distances yielding longer RTs.

\section{Method}

\section{Participants}

Twenty undergraduate and postgraduate students at Swansea University were paid $£ 4.00$ to participate in Experiment $2 \mathrm{~A}$, and a different sample of 20 undergraduate students from Swansea University was paid $£ 4.00$ to participate in Experiment $2 \mathrm{~B}$. All of the participants reported normal or corrected-to-normal vision, and none had participated in the previous experiments.

\section{Stimuli}

For both Experiments 2A and 2B, apart from the removal of the 3-D square, all remaining stimuli (fixation sphere, cue, and target) were exactly the same as those used in Experiments $1 \mathrm{~A}$ and 1B, respectively, with the only difference that now the object on which they appeared was removed and replaced by the same color as the background (black). Cues and targets appeared with the same perspective deformation that they appeared in during Experiments $1 \mathrm{~A}$ and $1 \mathrm{~B}$. Cue visibility against the black background $\left(0.22 \mathrm{~cd} / \mathrm{m}^{2}\right)$ was $C_{\mathrm{W}}=$ 31.81 for both rotated and unrotated displays.

\section{Design and Procedure}

The experimental design and procedure of Experiments 2A and 2B were identical to those of Experiments $1 \mathrm{~A}$ and $1 \mathrm{~B}$, respectively. Although no object was present, for the purpose of comparison, the conditions were labeled the same as those in Experiments 1A and $1 \mathrm{~B}$, respectively.

\section{Experiment 2A Results}

For the catch trials $(2.0 \%$ false alarms $)$, responses faster than $100 \mathrm{msec}(4.0 \%)$ and slower than $1,000 \mathrm{msec}(0.3 \%)$ were excluded from the analyses. The correct mean RT in each experimental condition is shown in Table 1. A 3 (validity: valid, invalid between planes, invalid within planes) $\times 3$ (target depth: unrotated, far, near) repeated measures ANOVA showed a significant main effect of validity $[F(2,38)=5.00, p=.01]$, a nonsignificant main effect of target depth $[F(2,38)=1.60, p>.05]$, and a significant interaction $[F(4,76)=5.85, p=.0001]$.

\section{Invalid RT Analysis}

All invalid trials for Experiment 2A were labeled in the same way as they were in Experiment 1A, for the purpose of comparison.

Between-planes shifts. For invalid horizontal RTs, there was a significant effect of target depth $[F(2,38)=$ $5.63, p=.007]$, with RTs in the unrotated condition being longer than both near-to-far $[t(19)=2.23, p=.038]$ and far-to-near $[t(19)=2.91, p=.009]$ RTs. As was expected, there were no differences between the far-to-near and near-to-far conditions [ $t(19)< \pm 1, p>.05]$.

Within-planes shifts. There was a significant effect of target depth on invalid within-planes RTs $[F(2,38)=6.02$, $p=.005]$. Near RTs were significantly longer than unrotated RTs $[t(19)=2.63, p=.016]$ and far RTs $[t(19)=$ $2.93, p=.009]$. Unrotated RTs were longer than far RTs $[t(19)=3.60, p=.004]$. 


\section{Valid RTs and Validity Effects Analysis}

The effect of target depth (unrotated, far, near) on valid RTs was not significant $[F(2,38)<1, p>.05]$. The validity effects are shown in Figure 2C. For the between-planes shifts, validity effects were significant in the unrotated condition $(M=30.6 \mathrm{msec}, S D=48.2)[t(19)=2.39, p=$ $.02]$ and in the near-to-far condition $(M=11.5 \mathrm{msec}, S D=$ 9.6) $[t(19)=2.67, p=.04]$ and marginally significant for the far-to-near condition ( $M=10.6 \mathrm{msec}, S D=8.6)$ $[t(19)=1.91, p=.06]$. For the within-planes shifts, all validity effects were significant [unrotated, $M=25.0 \mathrm{msec}$, $S D=45.9, t(19)=2.10, p=.05]$; near depth, $M=$ $43.3 \mathrm{msec}, S D=48.1, t(19)=3.80, p=.001$; far depth, $M=12.0 \mathrm{msec}, S D=24.0, t(19)=2.26, p=.04]$.

Between-planes shifts. The effect of target depth was significant for the validity effects $[F(2,38)=3.79, p=$ .03]. Planned comparisons showed that the validity effects were significantly larger in the unrotated than in the farto-near $[t(19)=2.10, p=.02]$ and near-to-far $[t(19)=$ $2.40, p=.03]$ conditions. There was no significant difference between far-to-near and near-to-far validity effects $(t< \pm 1, p>.05)$.

Within-planes shifts. The within-planes validity effects similarly revealed a significant effect of target depth $[F(2,38)=5.64, p=.007]$, whereby costs in the near condition were significantly larger than those in the unrotated $[t(19)=2.20, p=.03]$ and far $[t(19)=3.53, p=$ $.002]$ conditions. The unrotated condition validity effects were larger than the far condition validity effects $[t(19)=$ $2.30, p=.03]$.

\section{Experiment 2B Results}

Responses for the catch trials (1.1\% false alarms), and responses faster than $100 \mathrm{msec}(2.4 \%)$ and slower than $1,000 \mathrm{msec}(0.2 \%)$ were not included in the analyses. The data from 1 participant were removed because that participant's RTs were higher than $2 S D$ s from the group mean. The correct mean RT in each experimental condition is shown in Table 1. A 3 (validity: valid, invalid between planes, invalid within planes) $\times 3$ (target depth: unrotated, far, near) repeated measures ANOVA showed significant main effects of validity $[F(2,36)=30.07, p=.0001]$ and target depth $[F(2,36)=4.46, p=.02]$ and a significant interaction $[F(4,72)=11.43, p=.0001]$.

\section{Invalid RT Analysis}

All invalid trials for Experiment 2B were labeled in the same way as they were in Experiment 1B.

Between-planes shifts. For the invalid betweenplanes RTs, there was a significant effect of target depth $[F(2,36)=4.44, p=.02]$. The unrotated RTs were longer than both the far-to-near and the near-to-far RTs $[t(18)=$ $2.56, p=.02$, and $t(18)=3.09, p=.006$, respectively]. There was no difference between the far-to-near and the near-to-far RTs $[t(18)< \pm 1, p>.05]$.

Within-planes shifts. There was a significant effect of target depth on invalid within-planes RTs $[F(2,36)=$ $11.48, p=.0001]$. The near RTs were longer than the unrotated $[t(18)=3.02, p=.007]$ and the far $[t(18)=4.94$, $p=.0001]$ RTs. The unrotated RTs were not significantly different from the far RTs $[t(18)<1, p>.05]$.

\section{Valid RTs and Validity Effects Analysis}

The effect of target depth (unrotated, far, near) on valid RTs was significant $[F(2,36)=5.31, p=.01]$. The validity effects are shown in Figure 2D. For the between-planes shifts, all of the validity effects were significant [unrotated $M=45.3 \mathrm{msec}, S D=23.1, t(18)=8.55, p=.001$; far-to-near, $M=19.4 \mathrm{msec}, S D=18.8, t(18)=4.49, p=$ .0001 ; near-to-far, $M=16.1 \mathrm{msec}, S D=22.3, t(18)=$ $3.14, p=.006]$. The validity effects were also significant for all within-planes shifts [unrotated, $M=24.6 \mathrm{msec}$, $S D=26.4, t(18)=4.06, p=.001 ;$ near, $M=50.4 \mathrm{msec}$, $S D=34.2, t(18)=6.42, p=.0001] ;$ far, $M=11.1 \mathrm{msec}$, $S D=23.7, t(18)=2.2, p=.05]$.

Between-planes shifts. Target depth had a significant effect on validity effects $[F(2,36)=13.86, p=.0001]$. The unrotated validity effects were larger than those in the far-to-near and near-to-far conditions $[t(18)=4.90, p=$ .0001 , and $t(18)=4.71, p=.0001$, respectively], with no difference between the two latter conditions $[t(18)<$ $\pm 1, p>.05]$.

Within-planes shifts. There was a significant effect of target depth $[F(2,36)=10.69, p=.0001]$, whereby the validity effects in the near condition were significantly larger than those in the far $[t(18)=5.11, p=.0001]$ and unrotated $[t(18)=2.21, p=.04]$ conditions. Furthermore, the validity effects were significantly larger for the unrotated than for the far condition $[t(18)=2.27, p=.03]$.

\section{Experiments 2A and 2B Discussion}

In Experiments 2A and 2B, we used the same cue-target configurations as in Experiments 1A and 1B, respectively, to examine the pattern of validity effects as a function of cue-target distance without the presence of the object. We reasoned that any differences between the pattern of results in Experiments 1A and 1B (object present) and 2A and $2 \mathrm{~B}$ (object absent) would reveal the influence that a change in the perceptual organization of space imposed by the object in the former has on selective attention. Indeed, the pattern of results in Experiments 2A and 2B (validity effects influenced by cue-target distance) was drastically different from that in Experiments 1A and 1B (validity effects invariant to cue-target distance but sensitive to target location relative to the viewer). Below, we compare the validity effects between object-present and object-absent trials separately for the $y$-axis and $x$-axis rotations.

\section{Comparison Between Experiment $1 \mathrm{~A}$ and Experiment 2A ( $y$-Axis Object Rotation)}

If the pattern of results in Experiments $1 \mathrm{~A}$ and $1 \mathrm{~B}$ was due to the perceptual organization of space imposed by an underlying object representation, as opposed to the normal spatial distribution of attention across 2-D space, an interaction between cue-target distance and experiment is predicted. Cue-target distance should significantly influence validity effects in Experiments 2A and 2B (object absent) but not in Experiments 1A and 1B (object 
present). The following analyses confirm this prediction. Figures 3A-3C illustrate validity effects as a function of experiment and cue-target distance, separately for $y$-axis rotations (left panels) and $x$-axis rotations (right panels).

Between-planes shifts. A 2 (experiment: 1A, 2A) $\times$ 3 (target depth: unrotated [7.5० , far-to-near $\left[4.5^{\circ}\right]$, nearto-far $\left.\left[4.5^{\circ}\right]\right)$ mixed model ANOVA revealed a significant effect of target depth $[F(2,38)=4.02, p=.02]$, a nonsignificant main effect of experiment $[F(1,38)=2.64, p>$ $.05]$, and the expected significant interaction $[F(2,76)=$ $3.28, p=.04]$. Planned comparisons showed that the validity effects in the near-to-far condition were significantly smaller in Experiment 2A (object absent) than in Experiment $1 \mathrm{~A}$ (object present) $[t(38)=2.30, p=.04]$. There were no differences between experiments in the farto-near $[t(38)< \pm 1, p>.05]$ or unrotated $[t(38)=0.76$, $p>.05]$ conditions. Therefore, for between-planes attention shifts, cue-target distance linearly influenced RTs only when the object was absent but not when the object was present.

Within-planes shifts. A 2 (experiment: 1A, 2A) $\times$ 3 (target depth: unrotated $\left[7.5^{\circ}\right]$, far $\left[6.5^{\circ}\right]$, near $\left[8.5^{\circ}\right]$ ) mixed model ANOVA revealed a significant effect of target depth $[F(2,76)=4.07, p=.02]$. The effect of experiment was not significant $[F(1,38)<1, p>.05]$, but, critically, the predicted interaction was $[F(2,76)=3.58$, $p=.03]$. The validity effects in the far condition were significantly smaller for Experiment 2A than those for Experiment $1 \mathrm{~A}[t(38)=2.31, p=.02]$, with no betweenexperiments differences in the near $[t(38)< \pm 1, p>.05]$ or unrotated $[t(38)< \pm 1, p>.05]$ conditions.

\section{Comparison Between Experiment $1 \mathrm{~B}$ and Experiment 2B ( $x$-Axis Object Rotation)}

Between-planes shifts. A 2 (experiment: 1B, 2B] $\times$ 3 (target depth: unrotated $\left[7.5^{\circ}\right]$, far-to-near $\left[4.5^{\circ}\right]$, nearto-far $\left[4.5^{\circ}\right]$ ) mixed model ANOVA revealed a significant effect of target depth $[F(2,72)=11.18, p=.0001]$ and a nonsignificant effect of experiment $[F(1,36)=1.67$, $p>.05]$. As was expected, the interaction was significant $[F(2,72)=3.01, p=.05]$. Planned comparisons showed that the validity effects in the unrotated and the far-to-near conditions were significantly larger in Experiment 2B (object absent) than those in Experiment 1B (object present) $[t(36)=1.79, p=.04$, and $t(36)=1.73$, $p=.05$, respectively]. There were no differences between experiments in the near-to-far condition $[t(36)< \pm 1, p>$ $.05]$. Therefore, for the between-planes attention shifts, cue-target distance linearly influenced RTs only when the object was absent.

Within-planes shifts. A 2 (experiment: 1B, 2B) $\times$ 3 (target depth: unrotated $\left[7.5^{\circ}\right]$, far $\left[6.5^{\circ}\right]$, near $\left[8.5^{\circ}\right]$ ) mixed model ANOVA revealed a significant effect of target depth $[F(2,72)=5.60, p=.005]$ and a marginally significant main effect of experiment $[F(1,36)=3.66, p=$ $.06]$. Critically, the predicted interaction was significant $[F(2,72)=7.43, p=.001]$. The validity effects in the near condition were significantly larger for Experiment 2B (object absent) than those for Experiment 1B (object present) $[t(36)=3.18, p=.003]$, with no between-experiments differences in the far $[t(36)=1.62, p>.05]$ or unrotated $[t(36)=1.15, p>.05]$ conditions.

In summary, across the experiments reported here, 2-D cue-target distance linearly influenced RTs for both between- and within-planes attention shifts only when object contours were absent, not when the same distances were due to rotation of a 3-D object. Importantly, a comparison between the effect of target depth between Experiments $1 \mathrm{~A}$ and $1 \mathrm{~B}$ and $2 \mathrm{~A}$ and $2 \mathrm{~B}$ strengthens the findings of Experiments 1A and 1B. The fact that target depth had a significantly greater effect for Experiments $2 \mathrm{~A}$ and $2 \mathrm{~B}$ than for Experiments 1A and 1B argues that the addition of the object constrained how attention spread across space and that the null effect of target depth for withinplanes shifts in Experiments 1A and 1B was not due to insufficient power.

It is worth noting that the validity effects in Experiments $2 \mathrm{~A}$ and $2 \mathrm{~B}$ were modulated strictly by cue-target distance, despite the fact that cues and targets appeared with a perspective deformation, potentially indicating that they could be lying along an invisible surface extending in depth. That is, although this setup could have led the participants to interpret the cue-target relations as relations in depth (in particular, between-planes shifts), it did not, suggesting that the effects obtained in Experiments $1 \mathrm{~A}$ and $1 \mathrm{~B}$ were influenced by the presence of the object per se.

\section{GENERAL DISCUSSION}

In the present study, we examined the nature of representations of objects selected by object-based attention: Is depth explicitly encoded in the object representation, and if so, is it in viewer- or object-centered coordinates? When an object structured the scene (Experiments 1A and 1B), the 2-D cue-target distance was unrelated to the pattern of validity effects, but 3-D cue-target distance, as was imposed by the presence of the perceived 3-D square, clearly mattered. More specifically, within-planes far and near target validity effects did not differ, regardless of their differences in cue-target distance in 2-D space. At the same time, for between-planes shifts, although the 2-D distances in the near-to-far and far-to-near conditions were identical, the validity effects in those conditions were significantly different. Thus, attentional shifts were modulated by the location of the cue and target in depth, relative to the viewer. Furthermore, although 2-D cue-target distances in the unrotated and near-to-far conditions were different, the validity effects were equivalent. When the object, and the depth cues that it provided, were absent from the scene (Experiments $2 \mathrm{~A}$ and $2 \mathrm{~B}$ ), the validity effects were influenced by $2-\mathrm{D}$ cue-target distance, a finding previously observed in the literature (e.g., Downing \& Pinker, 1985; Egly \& Homa, 1991). This finding demonstrates that the pattern of validity effects observed when the object was present in Experiments 1A and $1 \mathrm{~B}$ was different from the way that attention spreads across 2-D empty space, indicating that the representation mediating validity effects in Experiments $1 \mathrm{~A}$ and $1 \mathrm{~B}$ was object based. 
A

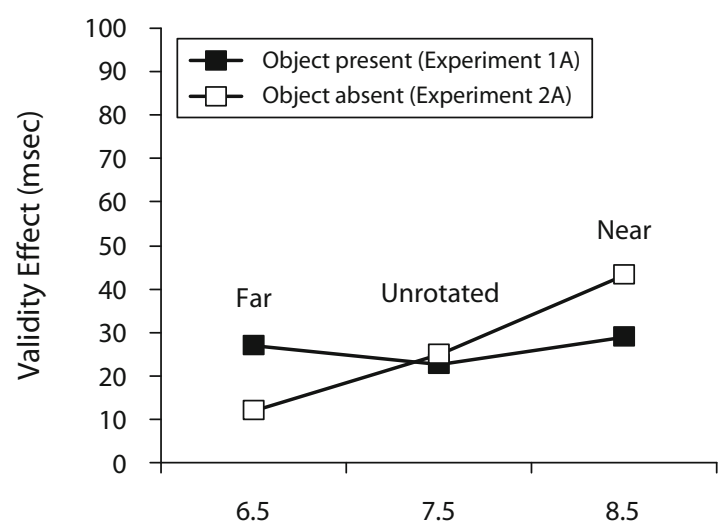

Cue-Target Distance
Within-Planes Shifts

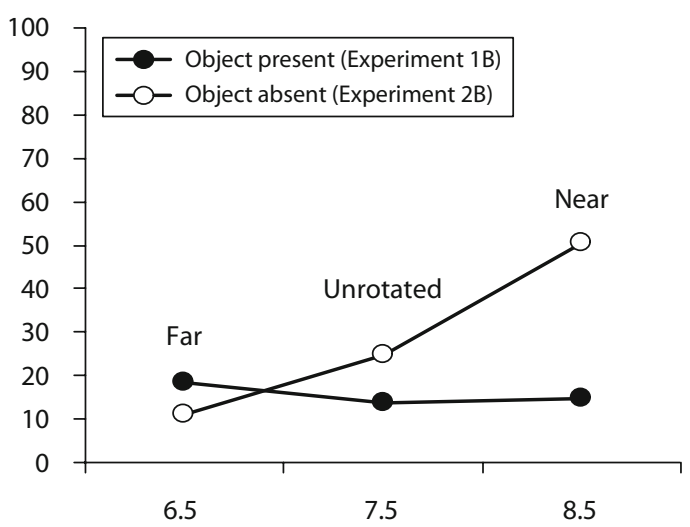

Cue-Target Distance
B

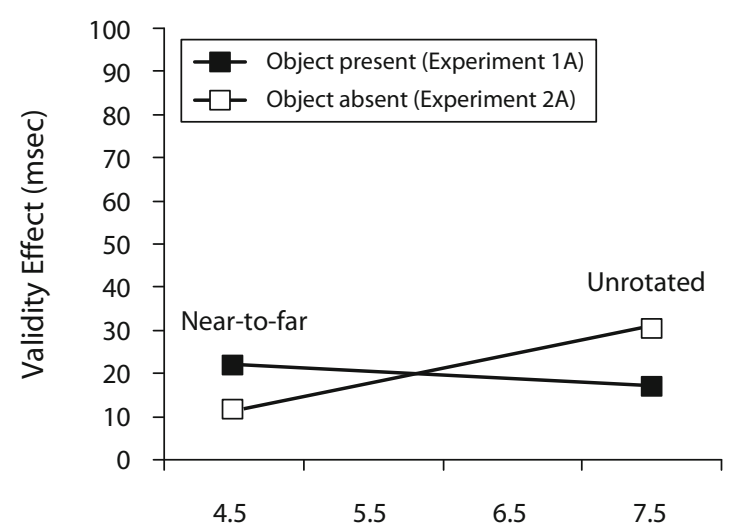

Cue-Target Distance

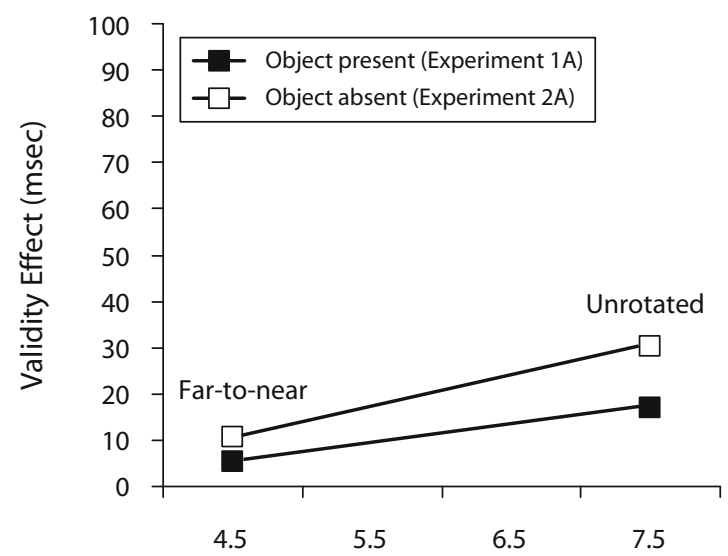

Cue-Target Distance

Between-Planes Shifts

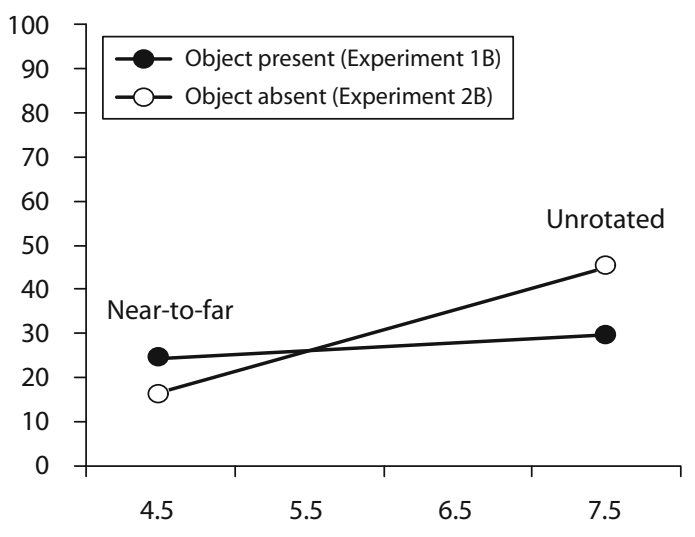

Cue-Target Distance

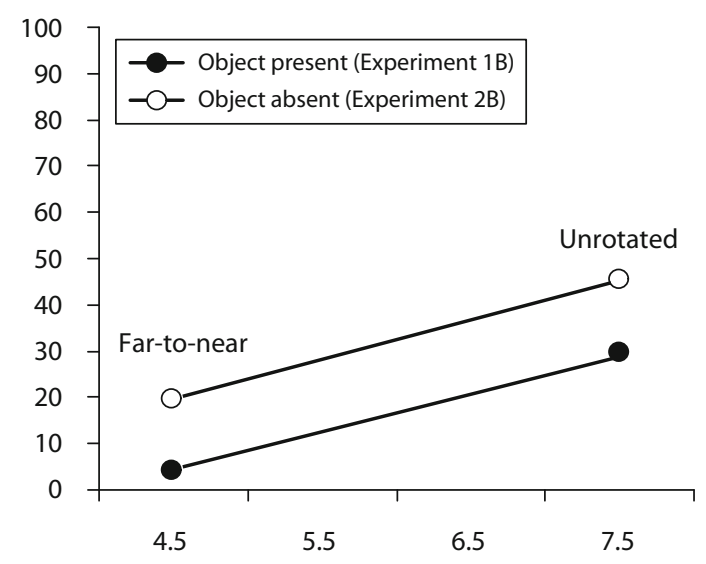

Cue-Target Distance

Figure 3. Experiment $\times$ cue-target distance (in degrees of visual angle) interaction in validity effects for within- and between-planes attention shifts. 
The present results are consistent with previous evidence that visual selective attention can be modulated by the perceived organization of the image (e.g., Duncan, 1984; Egly et al., 1994; Leek et al., 2003; Reppa \& Leek, 2003, 2006; Robertson \& Kim, 1999; Vecera et al., 2001; Vecera et al., 2000). The study by Robertson and Kim is particularly relevant here. By presenting a spatial cuing task superimposed over a scene with strong depth cues, they showed larger validity effects across a distant line that was perceived to be longer (the far corner of a room's wall) than a line perceived to be shorter (part of the near corner of the wall), despite the fact that the lines were identical in length. On the basis of this finding, they proposed that selective attention operated across space defined by the visual organization of the scene. However, in light of the present results, an equally valid interpretation of their data is that the walls used to structure the scene and alter the dynamics of selective attention evoked an object representation that explicitly encoded depth information. The perceptual illusion used by Robertson and Kim may have changed the representations mediating selective attention, just as the inclusion of the 3-D object in Experiments $1 \mathrm{~A}$ and $1 \mathrm{~B}$ altered the attentional dynamics relative to how attention shifted in unparsed space (Experiments $2 \mathrm{~A}$ and $2 \mathrm{~B}$ ).

The present findings demonstrate that attention spreads across a representation of a perceived 3-D object that encodes viewer-specific depth. Although viewer-centered representations (demonstrated by smaller validity effects when attention moves toward the viewer than when it moves away from the viewer) have previously been shown to mediate space-based attention (e.g., Andersen, 1990; Andersen \& Kramer, 1993; Arnott \& Shedden, 2000; Atchley \& Kramer, 2001; Downing \& Pinker, 1985; Gawryszewski et al., 1987) - that is, attention to distinct locations or objects in depth across space between objects - no study to our knowledge has previously shown that viewer-centered representations can also mediate object-based attention effects - that is, attention to locations within an object across space occupied by the object. Previous studies have provided evidence potentially compatible with both viewer- and object-centered representations (e.g., Gibson \& Egeth, 1994; Umiltà et al., 1995) by showing that attention is tied to a location within an object, irrespective of whether the stimulus location had changed relative to the viewer. Although these studies showed that selective attention operates on a representation that encodes the relationship between features of the object, they did not show whether their spatial relationship is encoded in 2-D or 3-D coordinates (i.e., whether depth is encoded as part of the object's representation), and did not exclude the possibility that those features are also encoded relative to the viewer (since they lacked manipulations in which attention shifts toward and away from the viewer). By manipulating the perceived orientation of the object in depth relative to the viewer, in Experiments $1 \mathrm{~A}$ and 1B, we dissociated the location of cues and targets relative to the object (which was the same across all invalid conditions and led to equivalent validity effects: unrotated $=$ far $=$ near) from their location relative to the viewer (which was different, depending on target depth condition, and led to different validity effects: far-to-near $<$ near-to-far). We found that object-based attention operated on a representation of the object that encoded cue-target locations in depth and that space within the object was coded in viewer-centered coordinates.

Although, in the present study, we examined the nature of object representations that mediate object-based attention effects, our findings also suggest that some properties are shared by object- and space-based attention. The present evidence for viewer-centered effects in shifts of attention within objects, together with previous evidence showing that attention in empty space (i.e., space between objects) operates in viewer-centered coordinates (e.g., Andersen, 1990; Andersen \& Kramer, 1993; Arnott \& Shedden, 2000; Downing \& Pinker, 1985; Gawryszewski et al., 1987), suggests a shared representational format for the two types of attention shifts. Additional evidence for this comes from a comparison of our study and that of Robertson and Kim (1999). Robertson and Kim proposed that attention operates on perceived space as structured by objects within a scene. Similarly, here we show that attention shifts within an object are constrained by the perceived space structured by the orientation of the object.

The present findings extend our knowledge about the nature of shape representations that mediate object-based attention, further sharpening our understanding of what constitutes an object in object-based attention. Previous evidence has shown that object-based attention operates on part-based representations of the selected objects (e.g., Leek et al., 2003; Reppa \& Leek, 2003, 2006; Vecera et al., 2001; Vecera et al., 2000). Here, we examined another neglected issue regarding the nature of the object representations that are selected in selective object-based attention: whether they encode depth. Incidentally, these issues - the nature of shape primitives and the coordinate frames of object representations - are the two most highly visible and debated in the object recognition research. What can the present findings tell us about the organizing shape primitives of the selected object representation? The withinplanes shift results (unrotated $=$ near $=$ far) and part of the between-planes shift results (unrotated $=$ near-to-far) suggest that the organizing primitives mediating object selection are not simply 2-D, thereby not fully supporting view-dependent theories of object recognition (e.g., Tarr \& Bülthoff, 1998; Tarr \& Pinker, 1989). Meanwhile, the difference between far-to-near and near-to-far shifts suggests that the primitives are also not fully 3-D, thereby also not entirely supporting view-independent theories of object recognition (e.g., Biederman, 1987; Marr \& Nishihara, 1978). If the underlying primitives were fully 3-D, there would presumably be no difference between the two types of shift. We therefore suggest that attention can operate on representations such as those described in Marr and Nishihara as a 2.5-D sketch representation, in which object surfaces are described in combination with their orientation in depth relative to the viewer.

The finding that object-based attention can operate on object representations that encode depth relative to the viewer also sets new constrains on hypotheses about the 
nature of representations that underlie object-based attention. For instance, Vecera and Farah (1994; see also Vecera, 1994) proposed that object-based attention effects could potentially reflect grouped-array effects-effects of attention in the space occupied by the object-without necessarily evoking an object-based representation (such as Marr and Nishihara's [1978] object-centered 3-D model representation). More recent work has further suggested that such 2-D grouped-array representations can be generated by the application of segmentation processes based on color or depth and can account for a number of objectbased attention findings (e.g., Müller \& O'Grady, 2009; O'Grady \& Müller, 2000). The present findings cannot easily be accounted for in terms of 2-D grouped-array representations and show that depth, apart from assisting figure-ground segmentation, must be encoded in object representations. Unless one assumes that a grouped-array representation makes explicit object surfaces and their orientation in depth relative to the viewer, our results are best accounted for in terms of an object-based attention mechanism that operates on a representation of the perceived 3-D object that encodes the object's orientation in depth relative to the viewer.

One concern with the present study is the possibility that the presence of an object in Experiments 1A and 1B might have lowered the visibility of cues relative to Experiments $2 \mathrm{~A}$ and $2 \mathrm{~B} .{ }^{2}$ This would predict smaller validity effects for Experiments 1A and 1B than for Experiments 2A and $2 \mathrm{~B}$, as well as longer mean RTs for Experiments 1A and 1B than for Experiments 2A and 2B. However, the data do not support either prediction. When we compared cuing effects across experiment and target depth (separately for Experiments 1A and 2A and for Experiments $1 \mathrm{~B}$ and 2B), we found no main effect of experiment for either comparison in either within-planes or between-planes shifts (all $p \mathrm{~s}>.05$ ). We also found no difference in mean RTs between Experiments 1A and 1B and Experiments 2A and 2B (all $p \mathrm{~s}>.05$ ). This suggests either that the presence of an object did not alter the effectiveness of our cues or that small differences in cue efficacy may have been present but did not significantly impact our data. Note that even if there were slight differences in cue efficacy not detectable by our study, such effects would not explain the different effects of rotation between Experiments 1A and 1B and Experiments 2A and 2B. Similarly, it is not possible to explain the lack of rotation effects in Experiments 1A and $1 \mathrm{~B}$ as being due to reduced cue efficacy. Note that rotation had no effect on validity effects when the object was present, regardless of whether rotation increased (for vertical far shifts) or decreased (for all other shifts) 2-D distance. In addition, since equivalent cues were used for Experiments 1A-2B, differences in the effect of target depth across study cannot be explained by cue visibility.

A final novel outcome of the present study concerns the finding that differences in shifting of attention across depth were induced using linear perspective alone. Previous observations of this anisotropy used binocular disparity cues, such as real or stereo-defined depth (e.g., Downing \& Pinker, 1985; Gawryszewski et al., 1987). The present study adds to this work with the finding that such viewer-centered effects can also be obtained by pictorial depth cues, therefore showing that the present findings are best accounted for by the operation of attention across a representation of 3-D objects that explicitly encodes viewer-based depth.

\section{AUTHOR NOTE}

We gratefully acknowledge the help of Hermann Müller and an anonymous reviewer for helpful comments on an earlier version of the manuscript. We thank E. Charles Leek and Olivia Cheung for helpful discussion and Lucy Kift and Kate Williams for their assistance in data collection. Correspondence concerning this article should be addressed to I. Reppa, Wales Institute for Cognitive Neuroscience, Department of Psychology, Swansea University, Singleton Park, Swanswa SA2 8PP, Wales (e-mail: i.reppa@swansea.ac.uk) or to D. Fougnie, Department of Psychology, Vanderbilt University, PMB 407817, 2301 Vanderbilt Place, Nashville, TN 97240-7817 (e-mail: d.fougnie@vanderbilt.edu).

\section{REFERENCES}

Andersen, G. J. (1990). Focused attention in three-dimensional space. Perception \& Psychophysics, 47, 112-120.

Andersen, G. J., \& Kramer, A. F. (1993). Limits of focused attention in three-dimensional space. Perception \& Psychophysics, 53, 658-667.

Arnott, S. R., \& Shedden, J. M. (2000). Attention switching in depth using random-dot autostereograms: Attention gradient asymmetries. Perception \& Psychophysics, 62, 1459-1473.

Atchley, P., \& Kramer, A. F. (2001). Object- and space-based attentional selection in three-dimensional space. Visual Cognition, 8, $1-32$

Atchley, P., Kramer, A. F., Andersen, G. J., \& Theeuwes, J. (1997). Spatial cuing in a stereoscopic display: Evidence for a "depth-aware" attentional focus. Psychonomic Bulletin \& Review, 4, 524-529.

BAYLIS, G. C., \& Driver, J. (1992). Visual parsing and response competition: The effect of grouping factors. Perception \& Psychophysics, 51, 145-162.

Biederman, I. (1987). Recognition-by-components: A theory of human image understanding. Psychological Review, 94, 115-147.

Biederman, I., \& Gerhardstein, P. C. (1995). Viewpoint-dependent mechanisms in visual object recognition: Reply to Tarr and Bülthoff (1995). Journal of Experimental Psychology: Human Perception \& Performance, 21, 1506-1514.

Bülthoff, H. H., Edelman, S. Y., \& Tarr, M. J. (1995). How are three-dimensional objects represented in the brain? Cerebral Cortex, 3, 247-260.

Downing, C., \& Pinker, S. (1985). The spatial structure of visual attention. In M. I. Posner \& O. S. M. Martin (Eds.), Attention and performance XI (pp. 171-187). Hillsdale, NJ: Erlbaum.

Duncan, J. (1984). Selective attention and the organization of visual information. Journal of Experimental Psychology: General, 113, 501517.

Egly, R., Driver, J., \& Rafal, R. D. (1994). Shifting visual attention between objects and locations: Evidence from normal and parietal lesion subjects. Journal of Experimental Psychology: General, 123, 161-177.

Egly, R., \& Homa, D. (1991). Reallocation of visual attention. Journal of Experimental Psychology: Human Perception \& Performance, 17, 142-159.

Gawryszewski, L. D. G., Riggio, L., Rizzolatti, G., \& Umiltà, C. (1987). Movements of attention in three spatial dimensions and the meaning of "neutral" cues. Neuropsychologia, 25, 19-29.

Gibson, B. S., \& EGETH, H. (1994). Inhibition of return to object-based and environment-based location. Perception \& Psychophysics, 55, 323-339.

He, Z. J., \& NaKaYAma, K. (1995). Visual attention to surfaces in threedimensional space. Proceedings of the National Academy of Sciences, 92, 11155-11159.

Leek, E. C., Reppa, I., \& Arguin, M. (2005). The structure of 3-D object shape representations: Evidence from whole-part matching. 
Journal of Experimental Psychology: Human Perception \& Performance, 31, 668-684.

LeEK, E. C., RePPA, I., \& TipPER, S. P. (2003). Inhibition of return for objects and locations in static displays. Perception \& Psychophysics, 65, 388-395.

MARR, D., \& Nishinara, H. K. (1978). Representation and recognition of the spatial organization of three-dimensional shapes. Proceedings of the Royal Society B, 200, 269-294.

Müller, H. J., \& O'Grady, R. B. (2009). Object-based selection operating on a spatial representation made salient by dimensional segmentation mechanisms: A re-investigation of Egly and Homa (1984). Psychological Research, 73, 271-286.

O'Grady, R. B., \& Müller, H. J. (2000). Object-based selection operates on a grouped array of locations. Perception \& Psychophysics, 62, 1655-1667.

PosNer, M. I. (1980). Orienting of attention. Quarterly Journal of Experimental Psychology, 32, 3-25.

REPPA, I., \& LEEK, E. C. (2003). The modulation of inhibition-of-return by object-internal structure: Implications for theories of object-based attentional selection. Psychonomic Bulletin \& Review, 10, 493-502.

RePPA, I., \& LEeK, E. C. (2006). Structure-based modulation of IOR is triggered by object internal but not occluding shape features. Quarterly Journal of Experimental Psychology, 59, 1857-1866.

Robertson, L. C., \& Kim, M.-S. (1999). Effects of perceived space on spatial attention. Psychological Science, 10, 76-79.

TARR, M. J., \& BÜLTHOFF, H. H. (1998). Image-based object recognition in man, monkey, and machine. Cognition, 67, 1-20.

TARr, M. J., \& PinKer, S. (1989). Mental rotation and orientationdependence in shape recognition. Cognitive Psychology, 21, 233-282.

Theeuwes, J., Atchley P., \& Kramer, A. F. (1998). Attention control within 3-D space. Journal of Experimental Psychology: Human Perception \& Performance, 24, 1476-1485.

Ullman, S. (1989). Aligning pictorial descriptions: An approach to object recognition. Cognition, 32, 193-254.

Umiltà, C., Castiello, U., Fontana, M., \& Vestri, A. (1995). Objectcentered orienting of attention. Visual Cognition, 2, 165-181.

Vecera, S. P. (1994). Grouped locations and object-based attention: Comment on Egly, Driver, and Rafal (1994). Journal of Experimental Psychology: General, 123, 316-320.
Vecera, S. P., Behrmann, M., \& FilapeK, J. C. (2001). Attending to the parts of a single object: Part-based selection limitations. Perception \& Psychophysics, 63, 308-321.

Vecera, S. P., Behrmann, M., \& McGoldrick, J. (2000). Selective attention to the parts of an object. Psychonomic Bulletin \& Review, 7, 301-308.

Vecera, S. P., \& Farah, M. J. (1994). Does visual attention select objects or locations? Journal of Experimental Psychology: General, 123, 146-160.

Wu, B., OoI, T. L., \& HE, Z. J. (2004). Perceiving distance accurately by a directional process of integrating ground information. Nature, 428, 73-77.

\section{NOTES}

1. Although Müller and O’Grady (2009) showed evidence to suggest that "object selection operates on a saliency representation of the visual display that is inherently two dimensional, rather than three dimensional" (p. 283), they also suggested (their note 5) that it may be possible that attention can operate on 3-D object representations and that such an assumption could be directly addressed by having objects undulate in and out of their depth planes. In the present study, we employed a variant of such a procedure.

2. We thank H. Müller for this suggestion. On the basis of this suggestion, we also compared the overall mean RTs between Experiments 1A and $2 \mathrm{~A}$ and Experiments $1 \mathrm{~B}$ and $2 \mathrm{~B}$ to determine whether the greater cue visibility when the object was absent led to overall shorter RTs than when the object was present. An independent-samples $t$ test revealed no significant difference between the mean RT for Experiment 1A ( $M=$ $322 \mathrm{msec}, S D=46.73)$ and that for Experiment $2 \mathrm{~A}(M=324 \mathrm{msec}$, $S D=42.02)[t(38)=-0.47, p>.05]$. There was also no significant difference between the mean RT for Experiment 1B $(M=349 \mathrm{msec}$, $S D=30.64)$ and that for Experiment $2 \mathrm{~B}(M=337 \mathrm{msec}, S D=46.23)$ $[t(36)=0.88, p<.05]$.

(Manuscript received September 4, 2009; revision accepted for publication January 8, 2010.) 